\title{
Healthcare Resource Consumption and Related Costs of Patients Estimated with Treatment-Resistant Depression in Italy
}

\author{
Valentina Perrone' \\ Diego Sangiorgi ${ }^{1}$ \\ Margherita Andretta ${ }^{2}$ \\ Giuseppe Ducci ${ }^{3}$ \\ Bruno Forti ${ }^{4}$ \\ Pier Cesare Francesa Morel $^{5}$ \\ Marco Gambera ${ }^{6}$ \\ Giuseppe Maina ${ }^{7}$ \\ Claudio Mencacci ${ }^{8}$ \\ Francesco Saverio Mennini ${ }^{9}, 10$ \\ Enrico Zanalda "I \\ Luca Degli Esposti (iD)
}

'CliCon S.r.l., Health, Economics \&

Outcomes Research, Bologna, Italy; ${ }^{2} \mathrm{UOC}$

Assistenza Farmaceutica Territoriale,

Azienda ULSS 8 Berica, Vicenza, Italy;

${ }^{3}$ Mental Health Department, ASL Roma I,

Rome, Italy; ${ }^{4}$ Mental Health Department -

Azienda ULSS n I "Dolomiti", Veneto

Region, Italy; 5 Janssen-Cilag SpA, Milano,

Italy; “"OSPEDALE P. PEDERZOLI" Casa di

Cura Privata S.p.A., Peschiera del Garda,

Verona, Italy; ${ }^{7}$ Department of

Neuroscience "Rita Levi Montalcini",

University of Turin, University Hospital San

Luigi Gonzaga, Turin, Italy; ${ }^{8}$ Department of

Neuroscience, ASST Fatebenefratelli Sacco,

Milan, Italy; ${ }^{9}$ EEHTA - CEIS (Centre for

Economic and International Studies),

Faculty of Economics, University of Rome

"Tor Vergata", Rome, Italy; ${ }^{10}$ Institute for

Leadership and Management in Health

Care, Kingston University, London, UK;

"'Department of Mental Health ASL TO3 \&

AOU San Luigi Gonzaga, Collegno, TO, Italy

Correspondence: Valentina Perrone

CliCon S.r.l. Health, Economics \&

Outcomes Research, via Murri, 9 ,

Bologna, 40 I37, Italy

Tel +39054438393

Fax +390544212699

Email valentina.perrone@clicon.it
Purpose: To analyse the healthcare resource consumption and related costs for the Italian National Health System of patients estimated to be affected by treatment-resistant depression (TRD) in Italy.

Patients and Methods: This was an observational retrospective study based on administrative databases, including those related to residential/semiresidential structures, of Veneto Region and the Local Health Unit of Bergamo in Italy (for a total of around 6 million healthassisted subjects). Between July 2011 and December 2017, all adult patients with a third antidepressant $(\mathrm{AD})$ after $\geq 2 \mathrm{AD}$ (each one with at least $\geq 4$ weeks duration, $\geq 1$ prescription at maximum dosage reported in datasheets, a grace period $\leq 30$ days when switching AD and treatment maintained $\geq 9$ months) were included. Overall and psychiatry-related healthcare resources consumption and related costs were estimated on a 12-months based analysis. Data were re-proportioned to the Italian population.

Results: We have previously estimated a total of 101,455 patients with TRD in Italy $(130,049$ considering the mean maximum dosage of $\mathrm{AD}$ ). Of them, $44.2 \%$ had at least a psychiatric hospitalization/visit or accessed a residential/semiresidential structure, and $31 \%$ added another $\mathrm{AD}$ or a mood stabilizer/antipsychotic drug. Patients with at least one psychiatryrelated hospitalization increased over the number of antidepressant lines from $12.0 \%$ during first line up to $24.5 \%$ during fourth line. Direct healthcare costs increased from $€ 4,405$ for first line to $€ 9,251$ from fifth line onwards. Psychiatry-related costs went from $€ 1,817$ (first line) to $€ 4,606$ (fifth line onwards) and were mainly driven by residential/semiresidential structures and hospitalizations.

Conclusion: An upward trend with number of AD lines was observed for all healthcare resource utilization and consequently for all direct costs, thus indicating an increasing burden for patients as they move forward AD lines.

Keywords: depression, healthcare costs, real-world, TRD

\section{Introduction}

Depressive disorders are regarded as a worldwide public health concern. ${ }^{1}$ Around one-third of patients suffering from major depression experience unsatisfactory responses to currently approved antidepressant drugs; the rates of unsuccessful treatments heavily contribute to the public health burden of this condition. ${ }^{2,3}$ Patients who fail to respond to a minimum of two different antidepressant treatments administered at an adequate dose for an adequate duration within the same depressive episode are considered to have treatment-resistant depression (TRD). ${ }^{4,5}$ According to the Italian guidelines, treatment strategies to manage TRD include the 
treatment optimization, ie, increase of antidepressant dosage or extension of the treatment duration; switch to another antidepressant belonging to the same or to a different class; augmentation with another antidepressant or with other drugs as antipsychotics or mood stabilizers. ${ }^{6}$

TRD has substantial effects on patients as well as on their families and society at large. ${ }^{7}$ It represents a humanistic and clinical burden since it has been associated with higher risk for comorbidity, increased suicidality, and significant impairment of cognitive function. ${ }^{8}$

TRD is considered as the major contributor to the economic burden of depression for patients and for Healthcare Systems. Indeed, several studies showed that, compared to major depressive disorder, TRD requires greater healthcare resource utilization. TRD patients are twice as likely to be hospitalized or to access outpatient and emergency room visits. ${ }^{7,8}$ Furthermore, TRD patients had higher direct and indirect medical costs than nontreatment-resistant major depressive ones. ${ }^{9}$

To the best of our knowledge, there is a lack of data regarding the economic burden of TRD in Italy. In a previous study, we estimated the number of patients affected by TRD in Italy by using selected criteria to identify TRD according to the European Medicines Agency (EMA) definition. ${ }^{10}$ The present study aims to provide an evaluation of the healthcare resource utilization and related costs of patients estimated to suffer from TRD in Italy.

\section{Methods}

This observational retrospective analysis was performed based on real-world data from Veneto Region and the Local Health Unit of Bergamo in Italy (approximately 6 million health-assisted subjects). Specifically, data were collected from administrative databases (beneficiaries, pharmaceuticals, hospitalization, outpatient specialist services) and databases of mental residential/semiresidential care facilities, ie, long stay treatment structures in which patients can settle in (residential stay) or spend part of the day (semiresidential stay). Data were re-proportioned on the Italian Population. The patient code in each database allowed electronic linkage between all different databases. All the results of the analyses were produced as aggregated summaries, which are not possible to assign, either directly or indirectly, to individual patients. According to Italian law, ${ }^{11}$ this study has been notified to and approved by the local Ethics Committee of the Region and LHU involved: specifically, the approval was obtained by the Ethics Committee "Comitato Etico per la sperimentazione clinica delle province di Verona e Rovigo", reference number 25368, approval date April, 26, 2018 and by the Ethics Committee "Comitato Etico di Bergamo", reference number 07/18, approval date January 30, 2018.

During July 1, 2011-December 31, 2017, all adult patients with TRD were included. TRD was identified by the presence of an antidepressant (ATC code N06A) line treatment after $\geq 2$ lines responding to the following criteria (Figure 1): ${ }^{10}$

- a duration of $\geq 4$ weeks with the same treatment since shorter duration could be due to safety issues or could indicate a different diagnosis other than major depression;

- the presence of at least one prescription for each antidepressant at the maximum dosage labeled in the summary of product characteristics (SmPC): the maximum dosage was chosen to discriminate between different diagnoses and to ensure the identification of patients with TRD for which a dose optimization/increment is suggested; ${ }^{6}$

- a grace period of $\leq 30$ days between lines: this criterion was selected in order to exclude, for instance, patients with well-being periods without treatment;

- if no changing occurs, treatment was maintained for at least 9 months, corresponding to the typical course

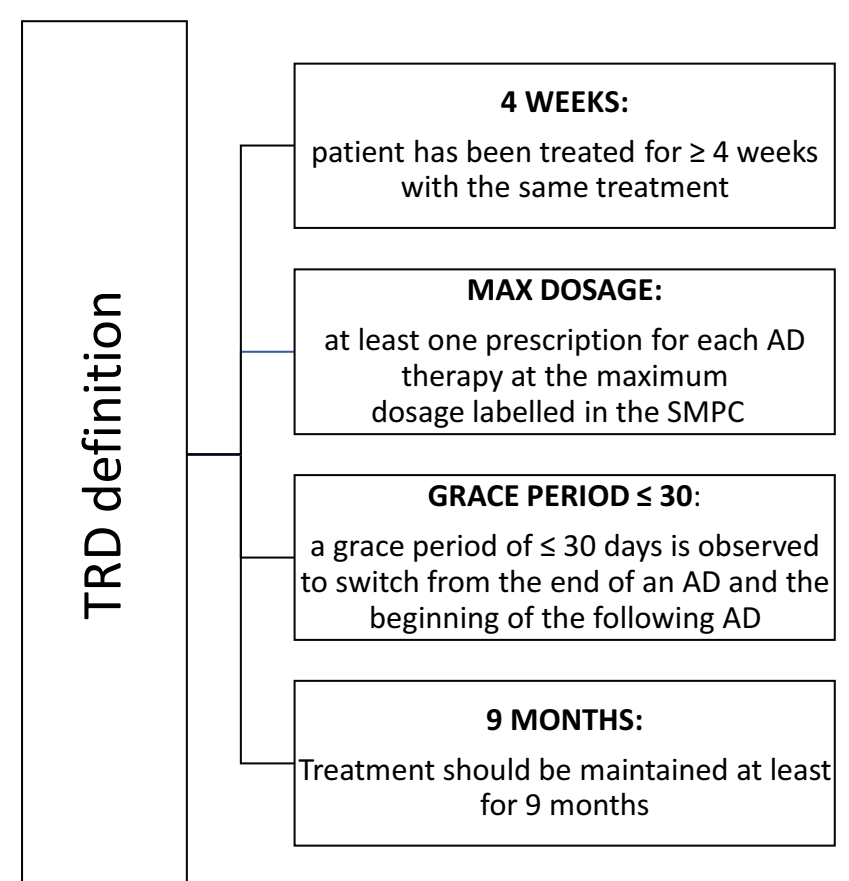

Figure I Criteria applied for the identification of TRD patients. 
of antidepressant treatment for a major depressive episode.

A treatment line was defined as the same antidepressant drug prescribed that met the four TRD criteria. Treatment lines could also be non-consecutive, ie, the presence of one or more antidepressant not matching TRD criteria between two antidepressant lines that met the four TRD criteria was allowed. Concomitant prescription of two antidepressants was considered as a single line. A switch from one antidepressant to another marked the beginning of a new line. The index date corresponded to the date of first antidepressant prescription during the inclusion period. Patients were followed-up starting from index date until death or December 31, 2017. Patients in treatment with antipsychotics (ATC code: N05A) or mood stabilizers (ATC code: N03A) in the 6-month period prior to index date were excluded.

Augmentation was evaluated during follow-up as the presence of antidepressant, mood stabilizers, or antipsychotics. Healthcare resource utilization was evaluated in terms of the presence of psychiatric-related visit (codes 94.12.1, 94.19.1), psychiatry-related hospitalizations (Major Diagnostic Categories 19; diagnosis-related group, DRG 425-433, 523), access to residential/semiresidential structures as number and percentage of patients using these resources among total patients identified. Costs analysis was performed from the perspective of the Italian National Health System (INHS) and comprised of drugs (antidepressants and other prescriptions), visits/tests (psychiatry-related and all-cause), psychiatry-related residential/semiresidential stays (as all costs for all services provided in these structures), and hospitalizations (psychiatry-related, all-cause). The price at the time of purchase for drug treatments was considered, while the costs for the hospitalizations were derived directly from DRGs codes; outpatient specialist visits costs were derived from regional tariffs. Mean annual costs for each resource was calculated as the sum of the cost for each resource between two consequent antidepressant lines divided by the sum of years between two consequent lines and divided by the total number of patients. Mean annual residential/semiresidential and psychiatry-related hospitalization costs were also calculated among patients in each antidepressant line and among patients that actually accessed into a structure/ hospital in each antidepressant line. A sensitivity analysis of healthcare costs was performed by considering a duration of $\geq 6$ weeks instead of a duration of $\geq 4$ weeks with the same treatment, in line with the literature. ${ }^{12,13}$

ANOVA test was used to compare mean annual costs. Statistical significance between antidepressant lines was accepted at $p<0.05$.

\section{Results}

As previously reported, by projecting data on the Italian population, 101,455 patients were estimated to be affected by TRD according to the inclusion/exclusion criteria applied and were included in the analysis. ${ }^{10}$ Of them, 63,842 were estimated in the age-range 18-64, and 37,613 were $\geq 65$ years old. Patients were prescribed at a mean dosage which represented $84.2 \%$ of the maximum dosage. When this proportion was considered as criterium, 130,049 patients were identified $(81,836$ in the age range $18-64$ and $48,213 \geq 65$ ).

Overall, $44.2 \%$ of TRD patients had at least a psychiatric hospitalization or visit or accessed a residential/semiresidential structure, and 31\% also required an augmentation. Patient's distribution according to psychiatric-related resources are reported in Figure 2.

B

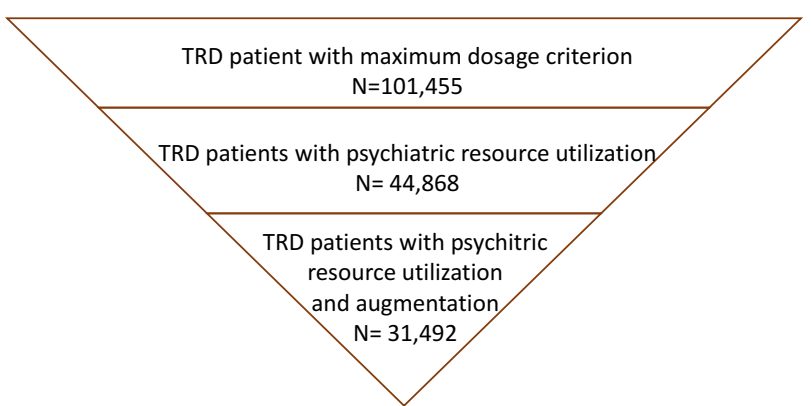

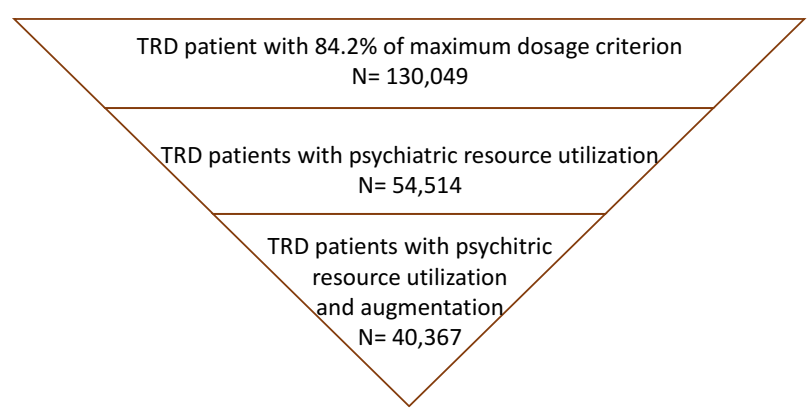

Figure 2 Italian projection of patient distribution according to psychiatric-related resource utilization considering (A) maximum dosage labelled in SMPC and (B) the ratio between mean dosage prescribed/maximum dosage labeled in SMPC. 


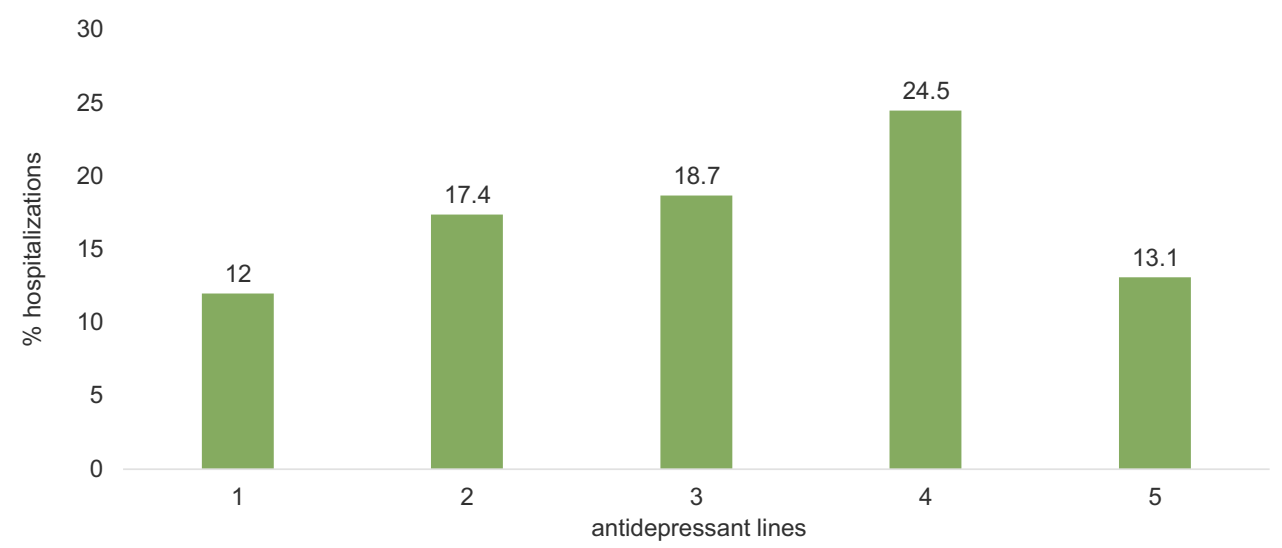

Figure 3 Psychiatry-related hospitalizations according to antidepressant lines.

The proportion of patients with at least one psychiatryrelated hospitalization increased over the number of antidepressant lines: $12.0 \%$ during the first line, $17.4 \%$ during the second one, $18.7 \%$ during the third one, up to $24.5 \%$ during the fourth line (Figure 3); $13.1 \%$ of patients with more antidepressant lines experienced at least a psychiatry-related hospitalization. However, this latter data must be interpreted considering that patients may not have ended the last line at the end of data availability, therefore it could be underestimated. Mean annual number of hospitalizations per patient ranged from 0.14 (first line) to 0.30 (fourth line).

A statistically significant increase of overall yearly costs from $€ 4,405$ for the first line to $€ 9,251$ for the fifth line onwards was observed $(p<0.001)$. All-cause hospitalizations were the major determinants of the total annual costs and went from $€ 1,345$ for the first line to $€ 2,582$ for the fifth line onwards $(p<0.001)$. The increments were particularly evident for total costs psychiatry-related, from $€ 1,817$ (first line) to $€ 4,606$ (fifth line onwards), as highlighted in red in Figure 4

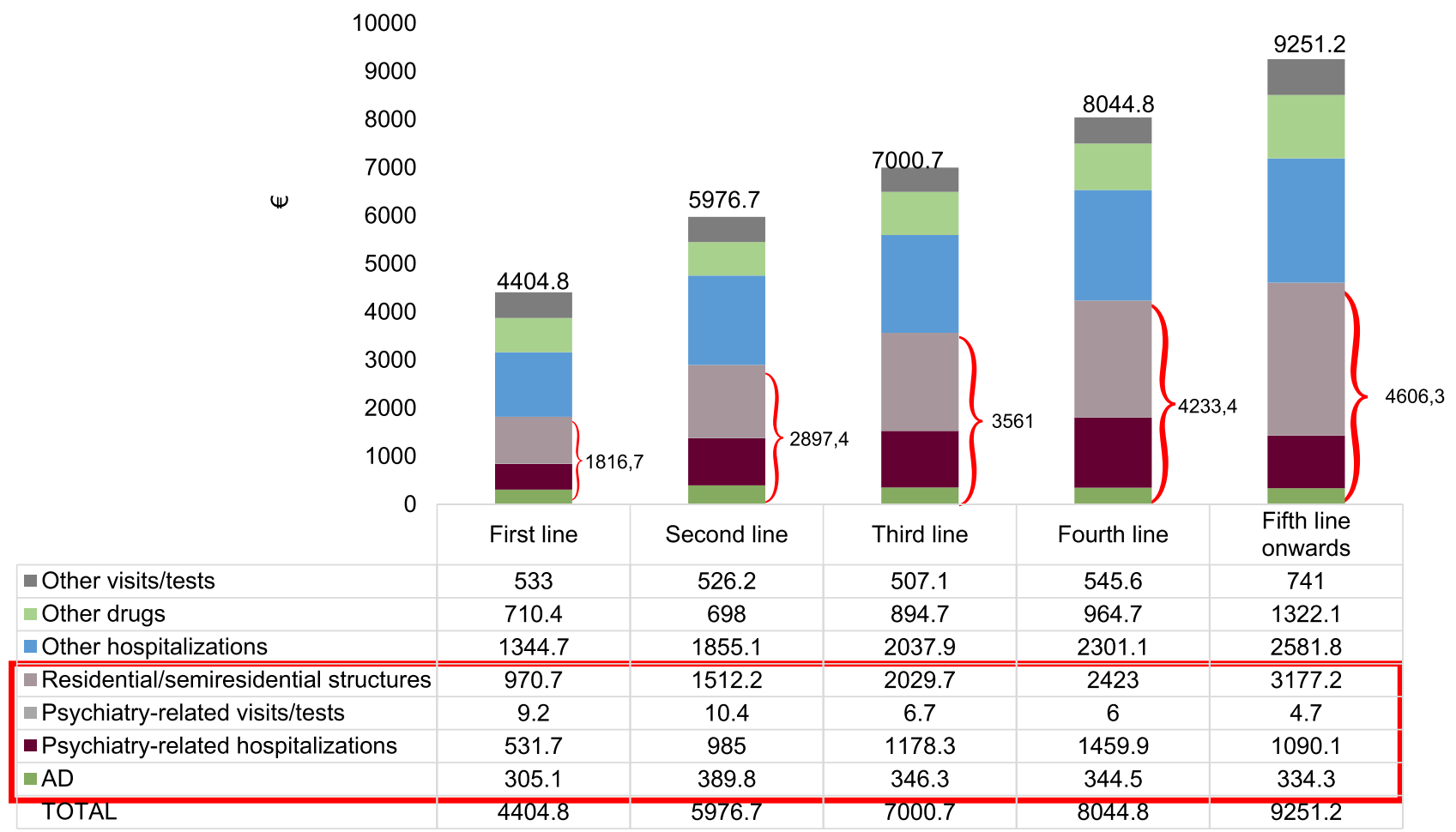

Figure 4 Mean annual all-cause and psychiatry-related costs according to antidepressant lines.

Notes: All comparisons were statistically significant $(p<0.001)$. The red box highlights the psychiatry-related costs. Overall psychiatry-related costs for each antidepressant line is reported next to red brackets. 


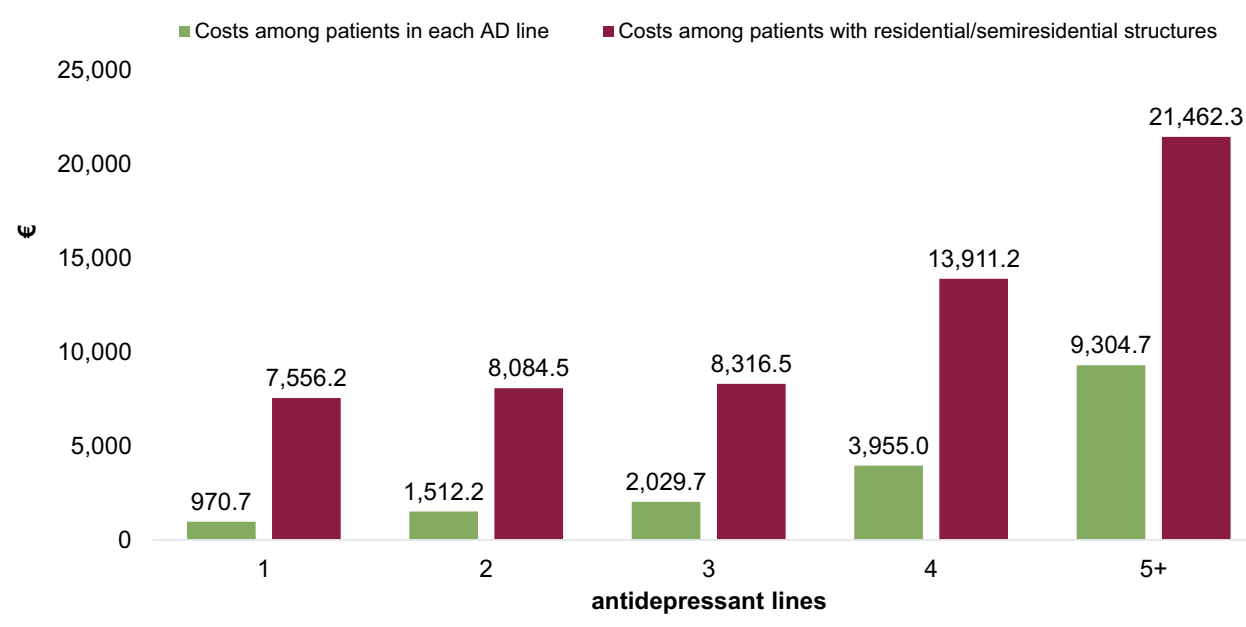

Figure 5 Mean annual costs of residential/semiresidential structures according to antidepressant lines.

Notes: All comparisons were statistically significant $(p<0.001)$. The number of patients within each line are reported in Supplementary Table I.

$(p<0.001)$. Psychiatry-related yearly costs were mainly driven by residential/semiresidential structures, that ranged from to $€ 971$ (first line) to $€ 3,177$ (fifth line onwards) $(p<0.001)$. A further analysis was performed to specifically evaluate the annual costs for residential/semiresidential structures and for psychiatry-related hospitalization per patient that actually was in each antidepressant line. Number/percentage for each line projected to the Italian population is reported in Supplementary Tables 1 and 2 . Among patients in each line, mean annual costs related to residential/semiresidential structure for TRD (starting from the third antidepressant line) increased from $€ 2,030$ for patients in the third antidepressant line to $€ 9,305$ for patients prescribed their fifth or more antidepressant line $(p<0.001)$ (Figure 5). The $34.1 \%$ of TRD patients identified were estimated to have accessed a residential/semiresidential structure. Costs were also evaluated among patients that actually accessed a residential/semiresidential structure stratified by antidepressant lines: in this case, the annual costs for these structures rose from $€ 7,556$ (first line) to $€ 21,462$ (fifth line onwards) $(p<0.001)$ and mean annual costs for residential/semiresidential structures was $€ 11,866$ per patient, and $€ 14,563$ per TRD patient (from third line onwards). Overall, $29 \%$ of patients had at least one access to hospital for psychiatry causes. Mean annual costs for TRD (starting from the third antidepressant line) related to psychiatry hospitalization for patients that actually had such hospitalization increased from $€ 6,301$ for patients in the third antidepressant line to $€ 8,321$ for patients prescribed their fifth or more antidepressant line $(p<0.001)$ (Figure 6), for a mean annual cost of $€ 6,135$ per hospitalized patient and $€ 6,860$ per hospitalized TRD patient (from line 3 onwards).

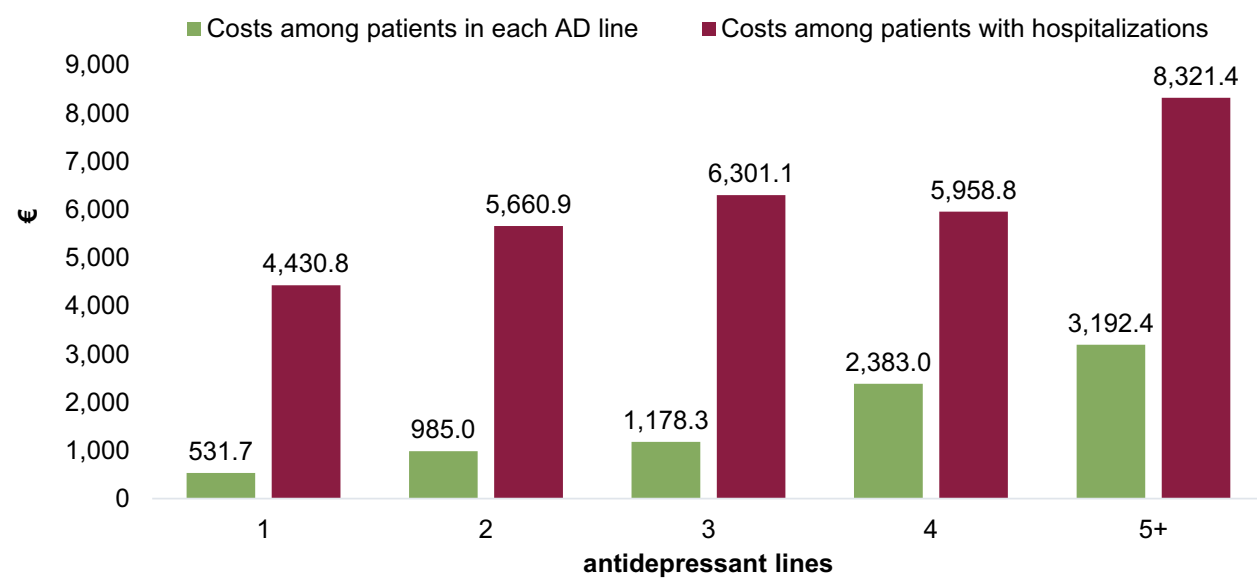

Figure 6 Mean annual costs of psychiatry-related hospitalizations according to antidepressant lines.

Notes: All comparisons were statistically significant $(p<0.001)$. The number of patients within each lines are reported in Supplementary Table 2. 
In the previous study, ${ }^{10}$ we observed that mean of lines duration exceeded the threshold of $\geq 4$ weeks, and that the estimation of TRD patients considering the treatment duration of $\geq 6$ weeks with the same antidepressant as criterion was 97,223 , ie, the overall sample changed in a negligible rate of patients. In the present study, we have calculated in a sensitivity analysis the healthcare costs for such patients (Supplementary Figures 1 and 2), obtaining similar values to those reported for the threshold of $\geq 4$ weeks.

\section{Discussion and Conclusion}

To date, few data are reported on TRD burden in Italy. In the present study, we provided an estimation of healthcare resource consumption and related costs per TRD patient for INHS in real-world settings of clinical practice. Our findings highlighted a growing trend of direct healthcare costs, both all-cause and psychiatry-related, with the increase in the number of antidepressant treatment lines.

In literature it has been widely reported that TRD patients incur more healthcare expenses and resource use compared to patient with non-TRD depressive syndromes, ${ }^{9,14}$ and the economic burden significantly increases with the level of resistance. ${ }^{13,15}$ This tendency was observed in our study as well, by comparing costs over different number of antidepressant lines. A possible explanation is given by the increment of morbidity during the depression state that require further treatments and leads to higher utilization of medical resource and subsequently to higher costs. ${ }^{15}$

Although drug therapy had a significant impact on the total expenditure, hospitalizations and residential/semiresidential structures were the major determinant of the overall costs. Given the lack of data on the economic impact of TRD in Italy, it is difficult to compare our results with international studies, given the different Healthcare Systems and different access to healthcare resources. However, several studies observed hospitalization costs to consistently increase disproportionately to other costs. ${ }^{15,16}$

Our cohort of patients reflected real clinical practice, and the results must be interpreted taking into account the limitations related to the observational nature of the study, which was based on data collected from administrative databases. First, there is not a consensus definition for TRD, therefore we could have over- or underestimated the number of TRD patients. Furthermore, out-of-pocket costs (ie, private psychiatric visits or private long-stay psychiatric structure) could not be evaluated as administrative databases contain data on healthcare resources reimbursed by INHS.
In conclusion, all healthcare costs estimated in the present analysis significantly increased with number of antidepressant lines, highlighting the greater need of resources utilization as patients move forward lines. The subsequent high economic impact further contributes to the overall burden of TRD.

\section{Acknowledgments}

The abstract of this paper was presented at the Virtual ISPOR European Congress 2020 (virtual) as a poster presentation with interim findings. The poster's abstract was published in Value in Health, 2020; 23: S587. https://doi. org/10.1016/j.jval.2020.08.1095.

\section{Funding}

This study was funded by Janssen Italy.

\section{Disclosure}

VP, DS, and LDE are employees of Clicon S.r.l., an independent company. The agreement signed by Clicon S.r.l. and Janssen Italy does not create any entityship, joint venture or any similar relationship between parties. Neither CliCon S.r.l. nor any of their representatives are employees of Janssen Italy for any purpose. PCFM is an employee of Hemar Department, Janssen Italy. GM has been a consultant/speaker for: Angelini, Boehringer, $\mathrm{Fb}$ Health, Innovapharma, Italfarmaco, Janssen, Otsuka, Lundbeck, Sanofi. EZ has been a consultant for Johnson \& Johnson, Otsuka, Lundbeck. All the remaining authors report no conflicts of interest.

\section{References}

1. World Health Organization. Depression and Other Common Mental Disorders: Global Health Estimates. World Health Organization; 2017. Licence: CC BY-NC-SA 3.0 IGO.

2. Cepeda MS, Reps J, Fife D, Blacketer C, Stang P, Ryan P. Finding treatment-resistant depression in real-world data: how a data-driven approach compares with expert-based heuristics. Depress Anxiety. 2018;35(3):220-228. doi:10.1002/da.22705

3. Marcatili M, Sala C, Dakanalis A, et al. Human induced pluripotent stem cells technology in treatment resistant depression: novel strategies and opportunities to unravel ketamine's fast-acting antidepressant mechanisms. Ther Adv Psychopharmacol. 2020;10:2045125320968331. doi:10.1177/2045125320968331

4. Murphy JA, Sarris J, Byrne GJ. A review of the conceptualisation and risk factors associated with treatment-resistant depression. Depress Res Treat. 2017;2017:1-10. doi:10.1155/2017/4176825

5. Clinical investigation of medicinal products in the treatment depression. European Medicines Agency; September 17, 2018. Available from: https://www.ema.europa.eu/en/clinical-investigationmedicinal-products-treatment-depression. Accessed September 18, 2019. 
6. Luchini F, Cosentino L, Pensabene L, Mauri M, Lattanzi L. Depressione resistente al trattamento: stato dell'arte Parte II. Trattamento. Riv Psichiatr. 2014;49(6):228-240. doi:10.1708/ 1766.19120

7. Gaynes BN, Asher G, Gartlehner G, et al. Definition of treatment-resistant depression in the medicare population. Agency for Healthcare Research and Quality (US); 2018. Available from: http:/www.ncbi.nlm.nih.gov/books/NBK526366/. Accessed September 18, 2019.

8. Jaffe DH, Rive B, Denee TR. The humanistic and economic burden of treatment-resistant depression in Europe: a cross-sectional study. BMC Psychiatry. 2019;19(1):247. doi:10.1186/s12888-019-2222-4

9. Li G, Zhang L, DiBernardo A, et al. A retrospective analysis to estimate the healthcare resource utilization and cost associated with treatment-resistant depression in commercially insured US patients. PLoS One. 2020;15(9):e0238843. doi:10.1371/journal.pone.0238843

10. Perrone V, Sangiorgi D, Andretta M, et al. Assessment of patients affected by treatment-resistant depression: findings from a Real-World Study in Italy. JPPD. 2020;04(03). doi:10.26502/ jppd.2572-519X0098

11. Agenzia Italiana del Farmaco (AIFA). Guideline for the classification and conduction of the observational studies on medicines; 2010. Available from: https:/www.agenziafarmaco.gov.it/ricclin/sites/ default/files/files_wysiwyg/files/CIRCULARS/Circular\%2031st\% 20May\%202010.pdf. Accessed June 18, 2021.
12. Ivanova JI, Birnbaum HG, Kidolezi Y, Subramanian G, Khan SA Stensland MD. Direct and indirect costs of employees with treatment-resistant and non-treatment-resistant major depressive disorder. Curr Med Res Opin. 2010;26(10):2475-2484. doi:10.1185/ 03007995.2010.517716

13. Amos TB, Tandon N, Lefebvre P, et al. Direct and indirect cost burden and change of employment status in treatment-resistant depression: a matched-Cohort Study using a US commercial claims database. J Clin Psychiatry. 2018;79(2):24-32. doi:10.4088/ JCP. $17 \mathrm{~m} 11725$

14. Zhdanava M, Kuvadia H, Joshi K, et al. Economic burden of treatment-resistant depression in privately insured US patients with co-occurring anxiety disorder and/or substance use disorder. Curr Med Res Opin. 2020:1-11. doi:10.1080/03007995.2020.1844645

15. Johnston KM, Powell LC, Anderson IM, Szabo S, Cline S. The burden of treatment-resistant depression: a systematic review of the economic and quality of life literature. $J$ Affect Disord. 2019;242:195-210. doi:10.1016/j.jad.2018.06.045

16. McIntyre RS, Millson B, Power GS. Burden of Treatment Resistant Depression (TRD) in patients with major depressive disorder in Ontario using Institute for Clinical Evaluative Sciences (ICES) databases: economic burden and healthcare resource utilization. $J$ Affect Disord. 2020;277:30-38. doi:10.1016/j.jad.2020.07.045
ClinicoEconomics and Outcomes Research

\section{Publish your work in this journal}

ClinicoEconomics and Outcomes Research is an international, peerreviewed open-access journal focusing on Health Technology Assessment, Pharmacoeconomics and Outcomes Research in the areas of diagnosis, medical devices, and clinical, surgical and pharmacological intervention. The economic impact of health policy and health systems

\section{Dovepress}

organization also constitute important areas of coverage. The manuscript management system is completely online and includes a very quick and fair peer-review system, which is all easy to use. Visit

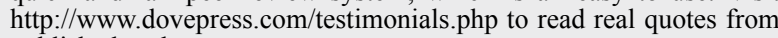
published authors. 